Schmerz 2010 $24: 309-312$

DOI 10.1007/s00482-010-0946-7

Online publiziert: 28. Juli 2010

(c) Deutsche Gesellschaft zum Studium des Schmerzes. Published by Springer

Medizin Verlag - all rights reserved 2010

H. Sorgatz ${ }^{1}$ C. Maier ${ }^{2}$

${ }^{1}$ Klinische Psychologie und Psychotherapie, Technische Universität Darmstadt

${ }^{2}$ Abt. Schmerztherapie, BG Universitätsklinikum Bergmannsheil, Ruhr-Universität Bochum

\title{
Einer neuen Wahrheit ist nichts schädlicher als ein alter Irrtum
}

\section{Übereinstimmungen neuer Leitlinien zur Opioidanwendung bei chronischen Schmerzen mit den Wirkprognosen der DGSS S3-Leitlinie LONTS}

Die DGSS S3-Leitlinie LONTS $[1,2]$ zur Langzeitanwendung von Opioiden bei nicht tumorbedingten Schmerzen wurde mit einem Methodenreport im Juni 2009 von der AWMF im Internet veröffentlicht. Links zu LONTS belegen seitdem die erste Seite bekannter Suchmaschinen; wohl auch, weil die Leitlinie seit Monaten Gegenstand hitziger und sehr kontroverser Debatten ist. Die Heftigkeit der Diskussion, auf die in diesem Editorial nicht eingegangen werden soll, unterstreicht die Wichtigkeit des Themas für die Schmerztherapeuten.

\section{Übereinstimmung mit neuen Leitlinien zur Opioidanwendung}

Leitliniengremien sind zur Eigenkontrolle verpflichtet. Bereits publizierte Leitlinien sollten kontinuierlich an später erschienenen angeglichen werden. In den letzten beiden Jahren haben neben der DGSS drei renommierte Schmerzgesellschaften sowie die kanadische National Opioid Use Guideline Group [3, 4, 5, 6] spezielle Leitlinien zur Opioidanwendung bei chronischen nicht tumorbedingten Schmerzen (CNTS) herausgegeben. Die 5 Leitli- nien unterscheiden sich teilweise in der Transparenz der Kriterien zur Erstellung klinischer Empfehlungen. Vorbildlich ist dabei die APS-Leitlinie über Chronic Opioid Therapy (COT), deren methodische Systematik und Problematik in einer Extrapublikation beschrieben sind [4, 7].

In der Wirkungsbeurteilung von Opioiden bei CNTS unterscheiden sich die 5 Leitlinien letztlich nur in der Diktion:

- APS-AAPM: „Although evidence is limited, an expert panel (...) concludes that COT can be an effective therapy for carefully selected and monitored patients with CNCP. However, opioids are also associated with potentially serious harms (...)“ ([4], p. 124).

- BPS: „There is evidence from clinical trials that opioids can be effective, in the short and medium term (...) However, the safety and efficacy of opioids in the long term is uncertain $(\ldots)^{\text {“ }}([6]$, p. 3$)$.

- ASIPP: „Opioids are commonly prescribed for chronic non-cancer pain and may be effective for short-term pain relief. However, long-term effec- tiveness of 6 months or longer is variable..." ([5], p. S5).

- NOUGG: „Opioids showed only small to moderate benefits for nociceptive (...) neuropathic (...) pain in improving function and relieving pain“ ([3], p. 16).

- DGSS: „Bewirken opioidhaltige Analgetika in einer Langzeitanwendung (3 Wochen bis 3 Monate) Schmerzlinderungen? Ja, jedoch gehen aus den Daten methodisch akzeptabler RCTs (randomized controlled trials) zur Anwendung von opioidhaltigen Analgetika mit einer Studiendauer von 3 Wochen bis 3 Monaten in der Regel nur klinisch schwache, wenngleich teilweise statistisch signifikante Schmerzlinderungen hervor“ und „Gibt es Belege für die analgetische Wirksamkeit von Opioiden bei einer Daueranwendung (länger als drei Monate bis mehrere Jahre)? Nein, nach umfangreichen systematischen Datenerhebungen an über 2400 Patienten mit CNTS in 10 un-

Beitragstitel: Zitat aus Johann Wolfgang von Goethe, Wilhelm Meisters Wanderjahre. 
kontrollierten Studien ist es bisher nicht gelungen, die Wirksamkeit opioidhaltiger Analgetika bei Anwendungszeiten von länger als drei Monaten mittels publizierter Daten aufzuzeigen." ([1], S. 2 ff).

Die Leitliniengremien halten somit eine analgetische Wirksamkeit von Opioiden bis zu einer Anwendungsdauer von ungefähr 3 Monaten wegen lückenhafter Nachweise lediglich für möglich. Die Übereinstimmung ist bemerkenswert, jedoch wegen gleicher Evidenzbasis nicht unerwartet. Die Leitlinien basieren auf einer Reihe von RCTs und Metaanalysen aus den letzten beiden Jahrzehnten. Daten aus kontrollierten klinischen Studien an mehr als 10.000 Patienten mit chronischem Schmerz und einer Studiendauer von bis zu 3 Monaten dienten als Entscheidungsgrundlage. Ergänzend wurden Ergebnisse unkontrollierter Studien mit länger dauernder Opioidanwendung bei mehr als 5000 Schmerzpatienten berücksichtigt.

\section{Unterschiede zwischen LONTS und anderen Leitlinien}

LONTS unterscheidet sich von den übrigen oben zitierten Leitlinien in zwei wesentlichen Aspekten:

1. Die qualitativ in allen Leitlinien gleiche Wirkungsaussage wird ergänzt durch eine aus RCTs abgeleitete quantitative Prognose, nach der mittlere Schmerzreduktionen von etwa 1o Einheiten auf einer 1ooer Schmerzskala auf Opioidwirkungen zurückgeführt werden können. Damit werden Klinikern Anhaltswerte für die Beratung ihrer Patienten zur Verfügung gestellt. Andere Leitlinien beschränken sich auf Aussagen wie „may be effective“, die uns für konkrete Therapieentscheidungen weniger geeignet schien.

2. Die Gremienentscheidungen für die LONTS-Leitlinie basieren zusätzlich auf systematisch analysierten Vergleichsdaten zu anderen Therapieoptionen bei CNTS. Erst dieses ermöglicht konkret formulierte Wirkungserwartungen für Opioidanwendungen im Vergleich mit an- deren Optionen. Gerade weil nichtopioidhaltige Analgetika in vergleichbaren Anwendungszeiten keine höheren Werte für Schmerzlinderung ergaben, erschien den LONTS-Gremien die abgeleitete Wirkungsannahme für opioidhaltige Analgetika vertretbar. Dies auch, da schwache Therapieoptionen in diesem Bereich der Schmerztherapie solange aufrechterhalten werden sollten, bis bessere zur Verfügung stehen [8].

\section{Langzeitanwendung von Opioiden - Mangel an Wirkung oder an publizierten Daten?}

Ein von allen Leitliniengremien erkanntes Problem sind die außerordentlich lückenhaften und selektiven numerischen Befunddarstellungen in Publikationen zur Langzeitanwendung von Opioiden über mehr als 3 Monate. Die daraus abgeleiteten vagen klinischen Hinweise in den Leitlinien sind Konsequenz eines erschreckenden Mangels an belastbaren Daten und damit nicht den Leitliniengremien zuzuschreiben. Trotz vieler Studien an mehreren Tausend Schmerzpatienten sind weder aus Einzelstudien noch aus Metaanalysen $[9,10]$ Daten herauszulesen, die auf eine analgetische Dauerwirkung schließen lassen, obwohl viele sog. Responder aus vorangegangenen, methodisch akzeptablen RCTs an solchen auf teilweise unbegrenzte Zeit angelegten Studien teilgenommen hatten. Die LONTSGremien konsentierten, dass trotz umfangreicher Datenerhebungen die publizierte Datenlage nicht auf eine Wirksamkeit opioidhaltiger Analgetika bei Anwendungszeiträumen von länger als 3 Monaten hinweist ([1], B3). Auch ein neueres Cochrane-Review kommt trotz ergebnisbeschönigender Maßnahmen zu keinem günstigeren Ergebnis: „The evidence regarding the effectiveness of long-term opioid therapy in CNCP is too sparse to draw firm conclusions, including quantity of mean of pain relief“ ([11], p. 26). Andere Non-RCT-Analysen von Patientendaten nach über 3-monatiger Opioidanwendung ergeben zudem Schmerzwerte von 58 bzw. 6o Einheiten auf einer 1ooer Schmerzskala [12, 13]. Dies ist nur 6 bis 8 Einheiten geringer als der durch- schnittliche Ausgangsschmerz unbehandelter Teilnehmer (66 Einheiten auf einer 1ooer Schmerzskala) aller analysierten RCTs und zeigt keinen klinisch relevanten Therapieerfolg an.

\section{Muss LONTS korrigiert werden?}

Zusammenfassend ergibt sich aus den aktuellen Leitlinien kein substanzieller Korrekturbedarf für das Leitliniengremium der DGSS. Im Gegenteil, die Kernaussagen von LONTS wurden durch andere Leitlinien bestätigt. Auch der erweiterte Ansatz von LONTS, Wirkungsprognosen quantitativ zu formulieren, und die von manchem als zu negativ bewerteten Aussagen über Opioide im Wirkungsvergleich mit anderen (ebenso schwach wirksamen) Therapieoptionen, wird durch keine neue Auswertung in Frage gestellt.

An dieser Stelle sei auf ein verbreitetes Missverständnis der Kritiker der LONTS-Leitlinie hingewiesen: Die vorsichtige und vermutlich realistische, aber dennoch positive Wirkungsannahme rechtfertigt einen Therapieversuch mit Opioiden. Aber dieser ist nicht mit der Wirkungszunahme gegenüber anderen Therapieoptionen zu begründen, sondern mit der individuellen Vorgeschichte und dem Risikoprofil des jeweiligen Patienten ([1], A3). Von daher wäre es absurd anzunehmen und inhaltlich wie juristisch nicht haltbar, aus den LONTSEmpfehlungen die Erstattungsfähigkeit von Opioiden bei chronischen Schmerzen zu bezweifeln. Denn diese ist nur von der Zulassung eines Medikaments abhängig. Ein u. U. regressbedrohter OffLabel-Use liegt nur dann vor, wenn andere als die zugelassene Indikation Anlass der Verschreibung sind. Alle in Deutschland legal erhältlichen oralen und transdermalen Opioide haben die Zulassung für mittlere oder starke Schmerzen je nach Art des Produkts.

\section{RCT - ein fragwürdiger Goldstandard?}

Nach dem Fundamentalsatz der Statistik, dass ein (noch) nicht bewiesener Unterschied keinen Beweis von Gleichheit darstellt, ist jede Erkennung auf Wirkungslosigkeit logisch problematisch und un- 
terliegt subjektiver Bewertung. Dennoch können jahrzehntelang unbefriedigend ausgegangene klinische Beobachtungen eine pragmatische Empfehlungsbasis schaffen. Dies um so mehr, als von Wirkungswerten unkontrollierter Studien noch die bis zu 60\%-ige Plazebowirkung abzuziehen ist. Bessere Studienkontrollen, ob in Form von RCTs oder als Direktvergleiche zwischen Medikamenten, führen in der Regel zu geringeren Effektstärken als unkontrollierte Vorher-Nachher-Beobachtungen einer Patientengruppe mit mehrmonatiger Opioidanwendung.

Fraglich bleibt jedoch, ob die Art der Evidenzermittlung aus RCTs zu Fehlschätzungen der Wirksamkeit einer Therapie führt. Objektivität, dazu zählen auch publizierte Daten aus RCTs, gilt als ein Merkmal der Evidenz, deren Interpretation jedoch grundsätzlich subjektiv ist [14]. Wir wissen von Fälschungen [15] der Abweichungen von den originalen Studienprotokollen $[16,17,18]$, von beachtlicher Transkription und Interpretation von Studiendaten in Metaanalysen sowie bestürzend niedrigen Interraterübereinstimmungen bei Reanalysen von Cochrane-Reviews bei der Beurteilung von RCTs und bei Metaanalysen $[17,19,20]$.

Der Verband forschender Arzneimittelhersteller hat in seinem kürzlich revidierten Positionspapier auf die verschiedenen Interessengruppen verwiesen, die in konzertierter - aber nicht notwendig abgesprochener - Weise erreichen, dass mit Literaturrecherchen „häufig kein getreues, umfassendes Abbild dessen, was tatsächlich (...) zu dem Thema erforscht worden ist" erhoben werden kann. Es werden eben „nicht alle Resultate aus klinischen Studien veröffentlicht" ([21], S. 1). Auch zeigt die jüngst im Deutschen Ärzteblatt veröffentlichte Metaanalyse [22] zum Einfluss des industriellen Sponsorings auf die Ergebnisdarstellung, dass dieser „publication bias“ bzw. „reporting bias" überwiegend zu einer Überschätzung der Wirksamkeit führt, wie dies am Beispiel der Antidepressiva und Gabapentin belegt wurde $[23,24]$. Folglich steht Leitlinienentwicklern lediglich eine Positivauswahl von Studienergebnissen zur Verfügung und $\mathrm{S}_{3}$-Leitlinien sind daher mit dem Generalverdacht eines eher positiven denn negativen Bias behaftet; so auch die Leitlinie LONTS.

Aber auch Unterschätzungen von Verumeffekten sind bei dieser Art der Evidenzgewinnung denkbar. RCTs erzeugen zwar allein durch ihre Durchführung eine artifizielle Situation mit einer Selektion von Patienten und Schaffung von insgesamt sehr günstigen Umgebungsbedingungen [25]. Schmerzpatienten mit vielen Komorbiditäten werden ebenso ausgeschlossen wie solche mit problematischer Mehrfachmedikation oder schlechter Compliance. De Williams hat 1999 schon gezeigt [26], wie diese Selektion und die Ablehnungsgründe der Probanden dazu führen, dass Patienten mit typischen Risikofaktoren für ein schlechteres Outcome selten in RCTs vorkommen. Anderseits verbessert die Teilnahme an einer RCT, wie Untersuchungen in der Kardiologie belegt haben, generell das Ergebnis unabhängig davon, ob ein Patient nun Verum oder Plazebo erhalten hat $[27,28]$. Dieses Phänomen ist verständlich, da bei doppelt verblindeten RCTs die günstigeren personellen Ressourcen sicherstellen, dass unabhängig von der Medikation auf jede Verschlechterung eines Patienten sofort reagiert wird. Effekte in Plazebogruppen können unter solchen Umständen besser ausfallen als in der täglichen Routine und damit zu einer Verkleinerung der Wirkungsunterschiede zwischen Plazebo und Verum führen.

Bei Analgetikastudien ist es kaum $\mathrm{zu}$ verhindern, dass Schmerzen von $\mathrm{Pa}$ tienten der Plazebogruppen durch unkontrollierte Selbstmedikation reduziert werden, während die Verumgruppenpatienten dieser nicht bedürfen. Die Höhe des bisher in RCTs und anderen Studien beobachteten, sich aus Plazebo- und Verumanteilen zusammensetzenden Gesamteffekts in den Verumgruppen bleibt davon jedoch unberührt. Vermutlich müssen zusätzlich zu RCTs vermehrt auch andere Studiendesigns verwendet werden, wie beispielsweise Kohortenstudien, die der Versorgungsrealität besser entsprechen und eine höhere externe Validität erreichen könnten als übliche RCTs $[25,29,30]$.

\section{Was bedeutet dies für die Praxis?}

Kritiker und Befürworter von LONTS sind sich in einem vermutlich einig: Leitlinien sind nicht dafür da, die Therapie des Einzelpatienten festzulegen oder eine bestimmte Therapieoption in jedem Fall auszuschließen. Leitlinien informieren Ärzte und somit indirekt auch Patienten über das gesicherte Wissen und insbesondere auch über Wirkungsprognosen für alternative Therapieoptionen. Der informierte Patient kann dadurch realistische Erwartungen entwickeln und Enttäuschungen vermeiden. Aus LONTS folgt, dass die Therapie von Arzt und Patienten engmaschig überwacht werden muss. Die Methoden hierzu sind bekannt. Möglicherweise werden zukünftig zumindest für bestimmte Indikationen deutlich wirksamere Analgetika zur Verfügung stehen. Bei anderen Krankheitsbildern, z. B. somatoformen Schmerzstörungen, könnte sich die Erkenntnis durchsetzen, dass der Einfluss von Opioiden und anderen Analgetika für den Therapieausgang unbedeutend ist.

Im Unterschied zur Leitlinie LONTS haben die 4 englischsprachigen Leitlinien ihren Schwerpunkt bei der Minimierung opioidbedingter gesundheitlicher und gesellschaftlicher Gefahren. Dieses unterstreicht im Einklang mit Versorgungsdaten aus Skandinavien [31] erneut, dass die gängigen Mythen, die immer noch ausschließlich als substanzorientierte Denk- und damit Erwartungsmuster formuliert werden, („Opioide sind das derzeit stärkste Schmerzmittel“ oder „Opioide sind immer wirksam“) nicht nur obsolet sind, sondern medizinische und gesellschaftliche Fehlentwicklungen begünstigen. Die internationale Diskussion über zunehmend häufiger zu behandelnde Suchterkrankungen durch legal verschriebene Opioide ohne erkennbare analgetische Wirksamkeit $[32,33,34,35$, 36] sind nur ein Beispiel für die immensen Gefahren einer irrationalen Opiattherapie. Somit dienen die aktuellen Leitlinien einschließlich der DGSS S3-Leitlinie LONTS auch der Prävention von allseits unerwünschten repressiven staatlichen Gegenmaßnahmen. Diese werden uns und unsere Patienten aber treffen, wenn der kritiklose Umgang mit Opioid- 
analgetika zur weiteren Verbreitung von Folgeerkrankungen führt, die das bislang in der Schmerztherapie Erreichte infrage stellen.

\section{Hachlo Sorgat}

H. Sorgatz
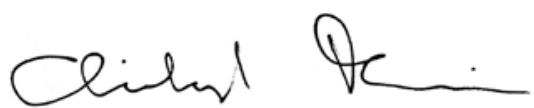

C. Maier

\section{Korrespondenzadresse \\ Prof. Dr. H. Sorgatz}

Klinische Psychologie und Psychotherapie, Technische Universität Darmstadt Alexanderstr. 10, 64283 Darmstadt sorgatz@psychologie.tu-darmstadt.de

Interessenkonflikt. Der korrespondierende Autor gibt an, dass kein Interessenkonflikt besteht.

\section{Literatur}

1. DGSS Leitlinien Kommission (2009) S3-Leitlinie Langzeitanwendung von Opioiden bei nicht tumorbedingten Schmerzen (LONTS). http://www. uni-duesseldorf.de/AWMF/ll/041-003.htm

2. Reinecke H, Sorgatz H (2009) S3-Leitlinie LONTS. Schmerz 23:440-447

3. National Opioid Use Guideline Group (2010) Canadian guideline for safe and effective use of opioids for chronic non-cancer pain. http://nationalpaincentre.mcmaster.ca/opioid/

4. Chou R, Fanciullo GJ, Fine PG et al (2009) Clinical guidelines for the use of chronic opioid therapy in chronic noncancer pain. J Pain 10(2):113-130

5. Trescot AM, Helm S, Hansen $\mathrm{H}$ et al (2008) Opioi$\mathrm{ds}$ in the management of chronic non-cancer pain: an update of American Society of the Interventional Pain Physicians' (ASIPP) guidelines. Pain Physician 11:S5-S62

6. BPS - The British Pain Society (2010) Opioids for persistent pain: good practice. http://www.britishpainsociety.org/book_opioid_main.pdf

7. Chou R, Ballantyne JC, Fanciullo GJ et al (2009) Research gaps on use of opioids for chronic noncancer pain: findings from a review of the evidence for an American Pain Society and American Academy of Pain Medicine Clinical Practice Guideline. J Pain 10(2):147-159

8. Dworkin RH, Turk DC, McDermott MP et al (2009) Interpreting the clinical importance of group differences in chronic pain clinical trials: IMMPACT recommendations. Pain 146(3):238-244

9. Trescot AM, Glaser SE, Hansen $\mathrm{H}$ et al (2008) Effectiveness of opioids in the treatment of chronic non-cancer-pain. Pain Physician 11:S181-S200
10. Noble M, Tregear SJ, Treadwell JR et al (2008) Long-term opioid therapy for chronic noncancer pain: a systematic review and meta-analysis of efficacy and safety. J Pain Symptom Manage 35(2):214-228

11. Noble M, Treadwell JR, Tregear SJ et al (2010) Long-term opioid management for chronic noncancer pain. Cochrane Database Syst Rev 20(1): CD006605

12. Dillie KS, Fleming MF, Mundt MP et al (2008) Quality of life associated with daily opioid therapy in a primary care chronic pain sample. J Am Board Fam Med 21(2):108-117

13. Sullivan MD, Von Korff M, Banta-Green c et al (2010) Problems and concerns of patients receiving chronic opioid therapy for chronic non-cancer pain. Pain 149(2):345-353

14. Miettinen OS (1998) Evidence in medicine: invited commentary. CMAJ 158(2):215-221

15. Ankier SI (2002) Dishonesty, misconduct and fraud in clinical research: an international problem. J Int Med Res 30:357-65

16. Chan AW, Hróbjartsson A, Haahr MT et al (2004) Empirical evidence for selective reporting of outcomes in randomized trials: comparison of protocols to published articles. JAMA 291(20):24572465

17. Kirkham JJ, Dwan KM, Altman DG et al (2010) The impact of outcome reporting bias in randomised controlled trials on a cohort of systematic reviews. BMJ 340:c365

18. Gøtzsche PC, Hróbjatsson A, Marić KL et al (2007) Data extraction errors in meta-analyses that use standardized mean differences. JAMA 298(4):430437

19. Ford AC, Guyatt Gh, Talley NJ et al (2010) Errors in the conduct of systematic reviews of pharmacological interventions for irritable bowel syndrome. Am J Gastroenterol 105(2):280-288

20. Tendal $B$, Higgins JP, Jüni $P$ (2009) Disagreements in meta-analyses using outcomes measured on continuous or rating scales: observer agreement study. BMJ 339:b3128-b3128

21. Verband forschender Arzneimittelhersteller (2010) Positionspapier „Publication bias“. http://www.vfa. de/download/SHOW/de/politik/positionen/pospublication-bias.html/pos-publication-bias.pdf

22. Schott G; Pachl H, Limbach U et al (2010) „Finanzierung von Arzneimittelstudien durch pharmazeutische Unternehmen und die Folgen " Dtsch Arztebl Int 107:279-85 und 107:295-301

23. Vedula SS, Bero L, Scherer RW, Dickersin K (2009) Outcome reporting in industry-sponsored trials of gabapentin for off-label use. N Eng J Med 361:1963-1971

24. Turner EH, Matthews AM, Linardatos E et al (2008) Selective publication of antidepressant trials and its influence on apparent efficacy. N Eng J Med 358:252-260

25. Black N (1996) Why we need observational studies to evaluate the effectiveness of health care. BM 312:1215-1218

26. De Williams AC, Nicholas MK, Richardson PH et al (1999) Generalizing from a controlled trial: the effects of patient preference versus randomization on the outcome of inpatient versus outpatient chronic pain management. Pain 83:57-65

27. Yuval R, Uziel K, Gordon N et al (2001) Perceived benefit after participating in positive or negative/ neutral heart failure trials: the patients' perspective. Eur J Heart Fail 3:217-23

28. Horwitz RI, Horwitz SM (1993) Adherence to treatment and health outcomes. Arch Intern Med 53:1863-1868
29. Chapman CR, Lipschitz DL, Angst MS et al (2010) Opioid pharmacotherapy for chronic non-cancer pain in the United States: a research guideline for developing an evidence-base. J Pain (in press)

30. Grootendorst DV, Jager KJ, Zoccali C et al (2010) Observational studies are complementary to randomized controlled trials. Nephron Clin Pract 114: c173-c177

31. Hamunen K, Paakkari P, Kalso E (2009) Trends in opioid consumption in the Nordic countries 20022006. Eur J Pain 13:954-962

32. Maier C (2008) Auch Sucht ist eine Krankheit. Schmerz 22:639-643

33. Ballantyne JC, LaForge KS (2007). Opioid dependence and addiction during opioid treatment of chronic pain. Pain 129:235-255

34. Fredheim OM, Skurtveit S, Breivik H et al (2010) Increasing use of opioids from 2004 to 2007 - Pharmacoepidemiological data from a complete national prescription database in Norway. Eur J Pain 14:289-94

35. Eriksen J, Sjøgren P, Bruera E et al (2006) Critical issues on opioids in chronic non-cancer pain: an epidemiological study. Pain 125:172-9

36. Cicero TJ, Lynskey M, Todorov A et al (2008) Comorbid pain and psychopathology in males and females admitted to treatment for opioid analgesic abuse. Pain 139:127-35 